\title{
Overview of the 63000 PWO Barrel Crystals for CMS ECAL Production
}

\author{
Etiennette Auffray
}

\begin{abstract}
In March 2007, the PWO crystal production for the barrel part of the CMS electromagnetic calorimeter has been completed. Since September 1998, 63000 crystals (61000 in Russia, 2000 in China) have been produced, received and tested in two regional centers (CERN and INFN Rome). This paper presents an overview of the procedures used from the $R \& D$ phase up to the final large scale reception and quality control. The crystals characteristics and the lessons learned from the production of this unprecedented amount of crystals in a HEP experiment are also presented.
\end{abstract}

Index Terms-CMS experiment, electromagnetic calorimeters, lead tungstate crystals (PWO).

\section{INTRODUCTION}

$\mathbf{T}$ HE Large Hadron Collider (LHC) is a particle accelerator that will allow exploring new physics at the highest energies ever achieved with accelerators. The LHC is currently being built at CERN and will be ready to produce collisions of protons at a centre of mass energy of $14 \mathrm{TeV}$ by the end of 2008 .

The Compact Muon Solenoid (CMS) [1], one of the four experiments at LHC, consists of a central inner tracker and calorimeters (electromagnetic and hadronic) imbedded in a 4 Tesla solenoid and a muon detection system outside the magnet. One of the main physics goals of CMS is the discovery of the Higgs boson. For a mass lower than $150 \mathrm{GeV}$, the cleanest channel is the Higgs decay in two photons, requiring an excellent energy resolution to distinguish the Higgs signal from the irreducible background. This has led CMS to the choice of a high resolution homogenous crystal electromagnetic calorimeter [2].

The CMS-ECAL is composed of 75848 Lead Tungstate crystals $\left(\mathrm{PbWO}_{4}\right.$, also called PWO), subdivided into a barrel (61200 crystals) and two end cap regions (7324 crystals each). In March 2007, the barrel production has been completed after 15 years of work. This paper presents results on the full production.

\section{HISTORY}

In 1992, at the first international conference on inorganic scintillators, Crystal 2000, in Chamonix, $\mathrm{PbWO}_{4}$ crystal for the first time raised attention for a potential use in high energy physics calorimetry [3] to [7]. Based on its high density $(8.3 \mathrm{~g} / \mathrm{cm} 3)$, its short radiation length Xo $(0.89 \mathrm{~cm})$, its fast light

Manuscript received June 27, 2007; revised September 11, 2007.

The author is with the CERN, Geneva 23, Switzerland (e-mail: etiennette. auffray@cern.ch).

Color versions of one or more of the figures in this paper are available online at http://ieeexplore.ieee.org.

Digital Object Identifier 10.1109/TNS.2007.913935
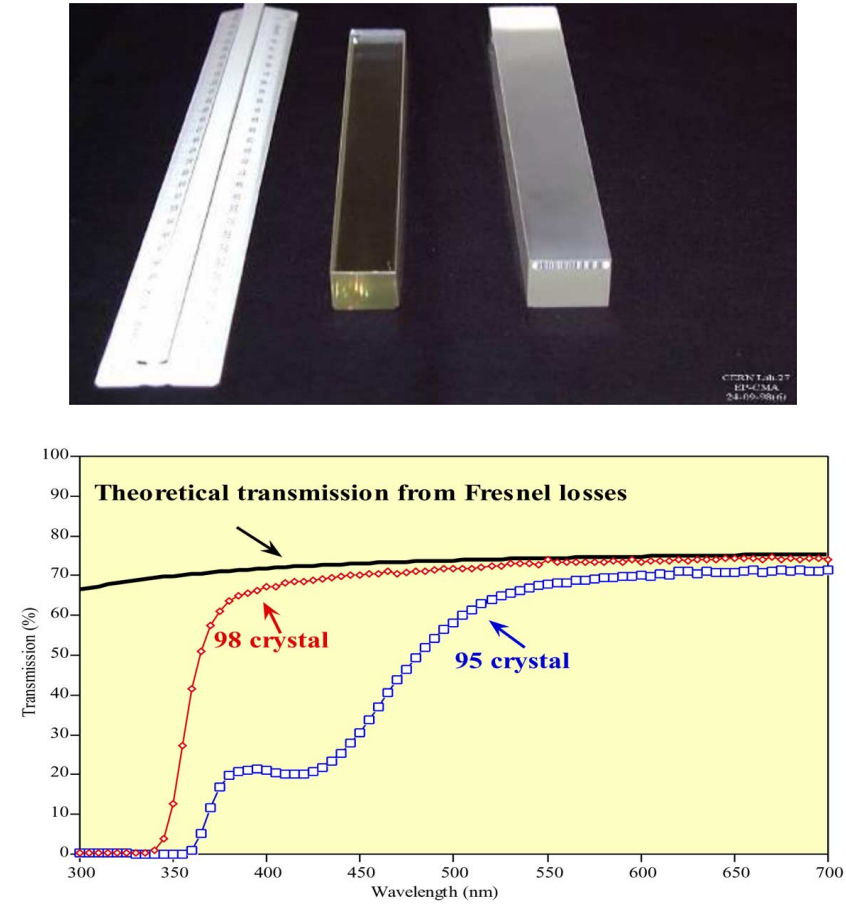

Fig. 1. (Top) Picture of crystal grown in 1994 and 1998. (Bottom) Improvement of optical transmission from 94 to 98 .

emission at $420 \mathrm{~nm}$ and the development of a new type of photodetectors (avalanche photodiode, APDs), providing a fast and compact way to read out crystals with relatively low light yield, PWO was chosen in 1994 to be used as scintillating material for CMS electromagnetic calorimeter

In consequence, a huge $R \& D$ effort was carried out in order to improve the scintillation characteristics of PWO: emission spectrum, light yield, decay time and radiation hardness [8]-[10].

Fig. 1 shows the optical transparency improvement obtained during this R\&D Phase.

In 1998, a pre-production phase started at the Bogoroditsk Techno-Chemical Plant (BTCP, Russia). 6000 crystals have been produced from 1998 to 2000 [11] to tune and optimise the growing process in order to allow the production of large size crystals $\left(\sim 2.5 \times 2.5 \times 23 \mathrm{~cm}^{3}\right)$ at an industrial scale (about 1000 crystals per month).

Based on this work, the production of the CMS barrel crystals could start in BTCP in 2001.

In 2005, an additional crystal production has started at the Shanghai Institute of Ceramic (SIC, China).

The two producers use a different growing technique: BTCP is using Czochrasky method while SIC is using the Bridgman method. 
TABLE I

GeOMETRY AND SuRface QuALITy SPECIFICATIONS

\begin{tabular}{ll}
\hline Parameters & Specification values \\
\hline \hline Dimensional Tolerance & $+0 \mathrm{~mm},-0.1 \mathrm{~mm}$ \\
Surface Flatness & $<0.02 \mathrm{~mm}$ \\
Chamfers & $-0.3-0.7 \mathrm{~mm}$ \\
Surface Roughness & For depolished Face $\sim 0.3 \mu \mathrm{m}$
\end{tabular}
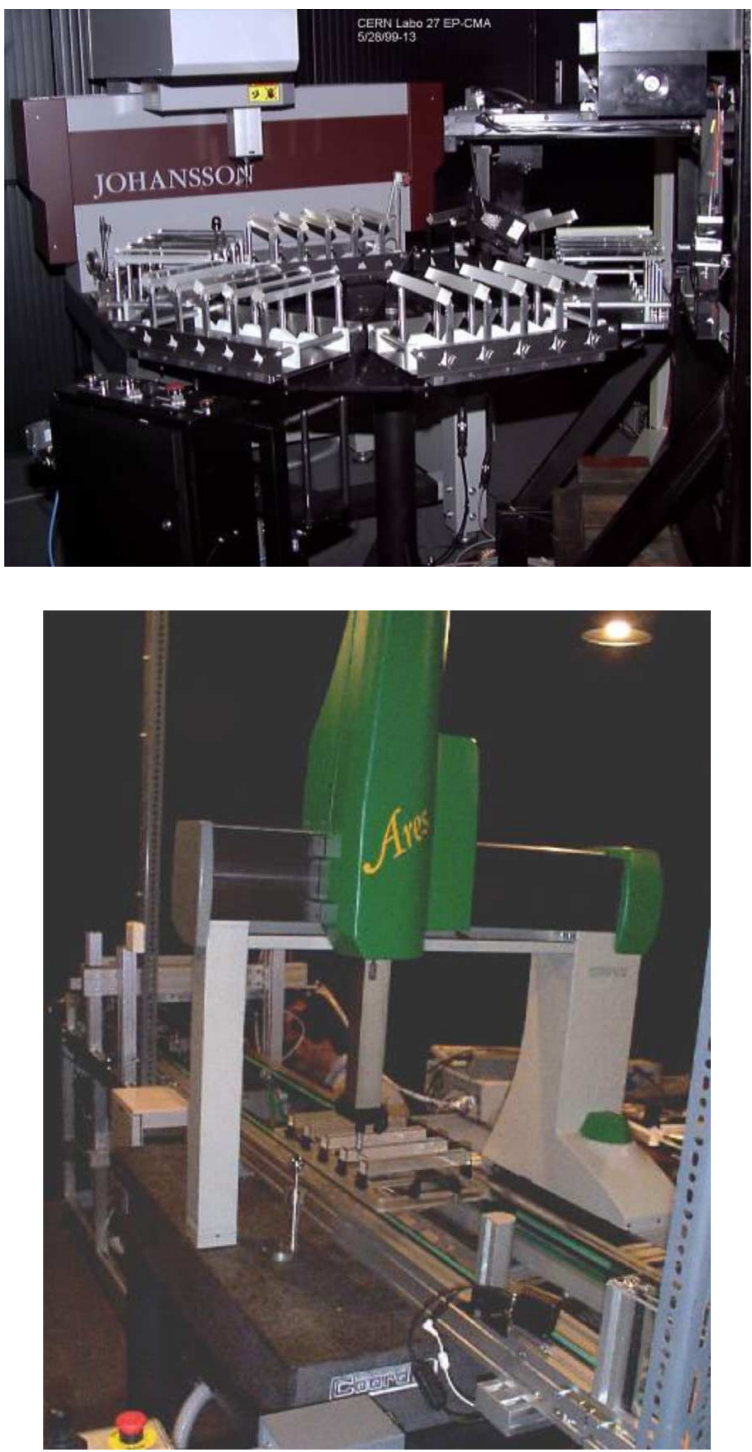

Fig. 2. (Top) View of the ACCOS system at CERN (ACCOCE) at (bottom) INFN/ENEA Rome regional center (ACCOR).

In March 2007, the full barrel crystals production was completed. A total of 61335 crystals were produced in BTCP from September 1998 to March 2007 and 1825 crystals from June 2005 to March 2007 in the SIC institute.

\section{QUALITY CONTROL}

To reach a very high calorimeter performance, the crystals must fulfill a set of specifications on dimensions and on different optical parameters [12]. In both regional centers at CERN and at Rome an automatic machine (ACCOCe and ACCOR machines)
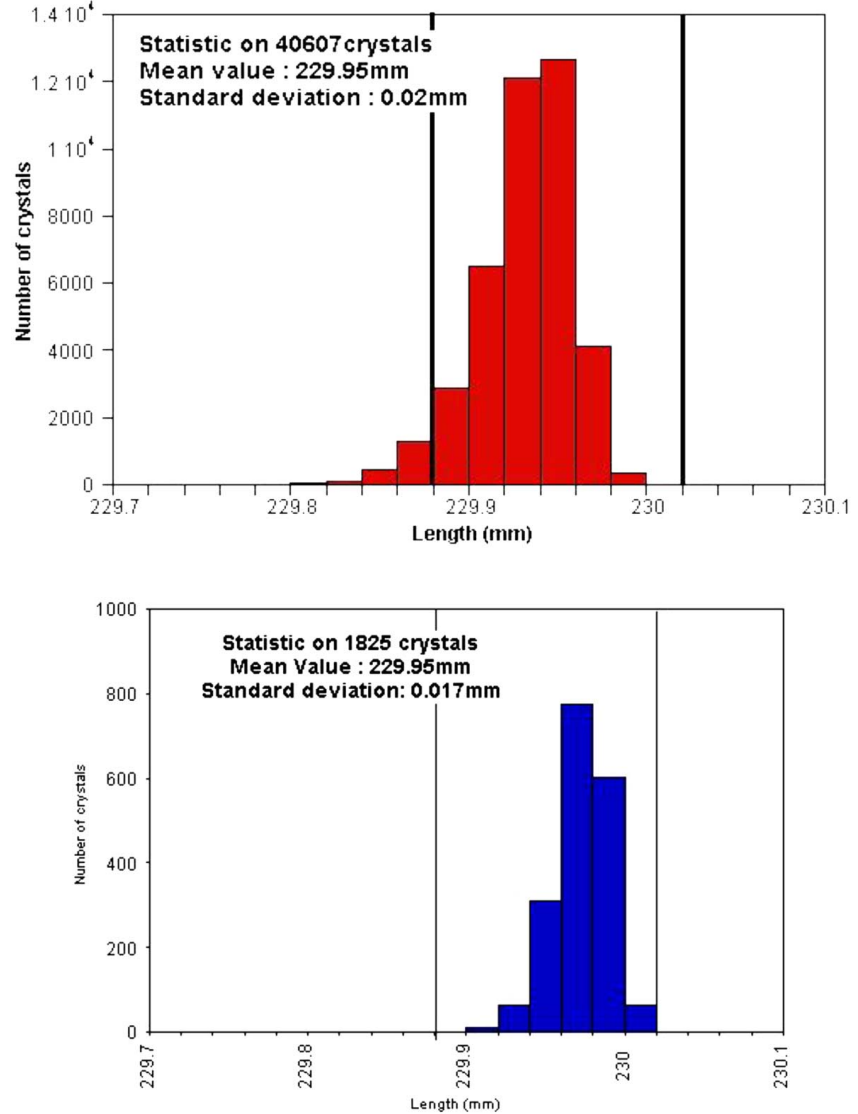

Fig. 3. Distribution of the crystal length measured on (top) BTCP and (bottom) SIC crystals.

was especially designed to check the specifications on each received crystal. A detailed description of their operation principles can be obtained from [13], [14]. Two CERN machines were also installed at BTCP and a Rome machine at SIC.

Each crystal was first checked at the production site and then, at its reception in regional centers, passed through the ACCOS machines for a full check of dimensions, transparency, light yield and light collection uniformity before being used in the assembly. Fig. 2 (top) shows a picture of ACCOCe at CERN. Fig. 2 (bottom) shows a picture the ACCOR machine at Rome.

Both machines were intecalibrated and the results obtained with ACCOCe and ACCOR were in a very good agreement [15].

\section{Results on Mass Production CRystals}

All crystals have to match specifications [12] for

- Geometry and Surface Quality;

- Light Yield and Light Collection Uniformity;

- Optical Transmission;

- Radiation Tolerance. 
TABLE II

LIGHT YIELD AND LIGHT COLLECTION UNIFORMITY SPECIFICATIONS

\begin{tabular}{ll}
\hline Parameters & Specification values \\
\hline \hline Light Yield (LY) @ 8Xo $(7.5 \mathrm{~cm})$ from front side of the & $\geq 8$ p.e./MeV \\
crystals & \\
Front Non Uniformity factor (FNUF) in the region of & $-0.35 \% / \mathrm{X} 0 \leq \mathrm{FNUF} \leq+0.35 \% / \mathrm{X} 0$ \\
maximal energy deposit &
\end{tabular}

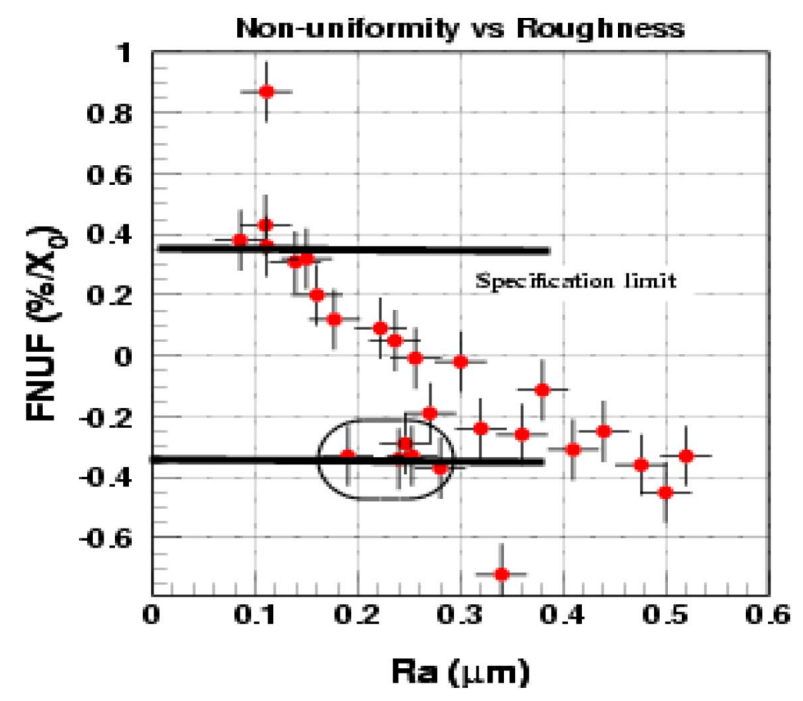

Fig. 4. Variation of the front light collection non uniformity (FNUF) with the roughness of the depolished face.

\section{A. Geometry and Surface Quality}

In order to implement a pointing geometry for the electromagnetic calorimeter, 17 different crystal geometry types were necessary for the barrel part of the calorimeter. [2] The detailed specifications for the crystal geometrical parameters and surface quality are listed in Table I.

Whereas chamfers and surface roughness are measured on a randomly chosen subsample, crystal dimensions and surface flatness are measured for each crystal using a sensor installed in the Accos machines. As an example, Fig. 3 shows the distribution of the crystal length for BTCP and SIC crystals separately, showing a spread of about $20 \mu \mathrm{m}$ for both producers.

\section{B. Light Yield and Light Collection Uniformity}

The detailed specifications for light yield and light collection uniformity are listed in Table II.

The light yield (LY) is measured using a radioactive source positioned at $7.5 \mathrm{~cm}\left(\sim 8 X_{0}\right)$ from the small end of the crystal with a photomultiplier of type XP2262B fully covering the rear face of the crystal, using silicon optical grease $(n=1.5)$ and with an integration time of $100 \mathrm{~ns}$. This position corresponds to the shower maximum for an incoming photon or electron of 50-100 GeV energy.

In order to avoid a degradation of the energy resolution due to longitudinal shower fluctuations, the light yield must remain constant along the crystal axis between $3 X_{0}$ and $15 X_{0}$ distance from the crystal front face. This property, expressed in terms of
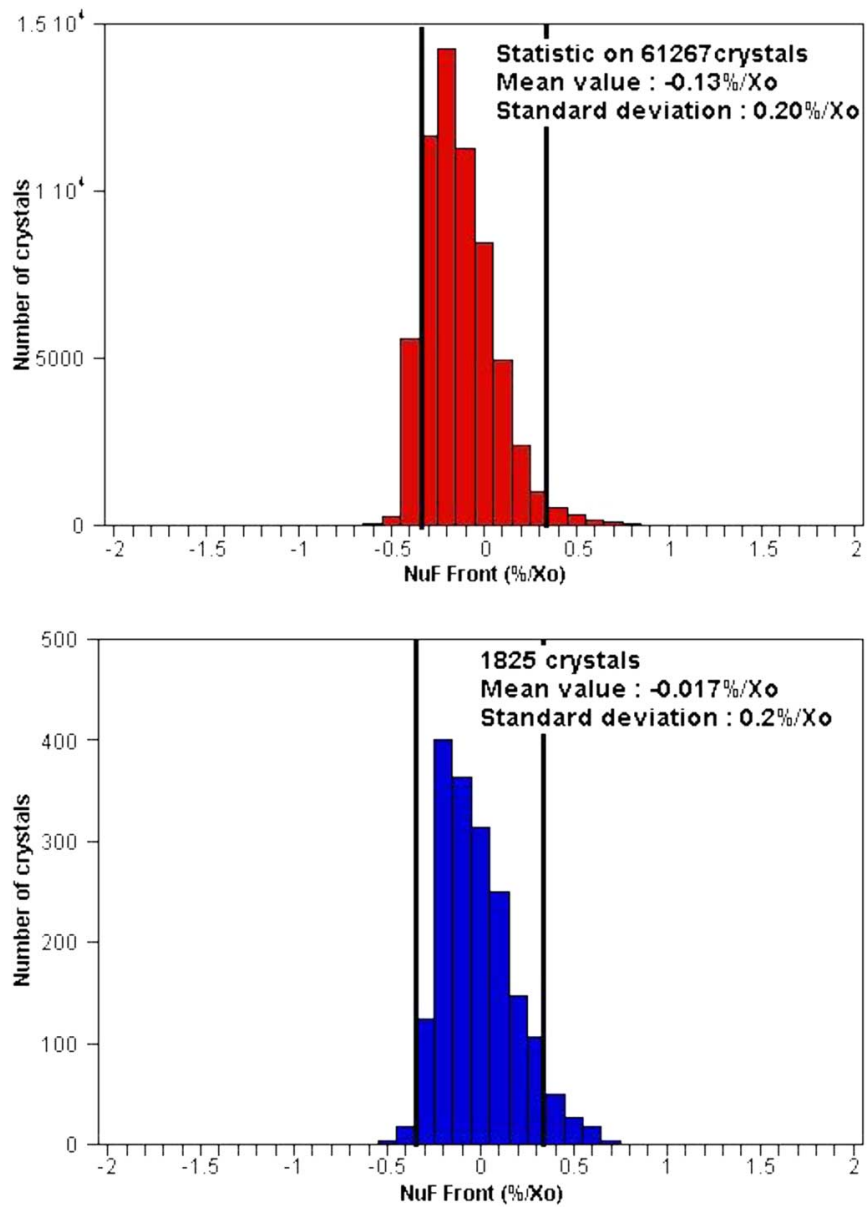

Fig. 5. Distribution of the front non uniformity for (top) BTCP (bottom) SIC crystals.

the relative LY variation per $X_{0}$ (FNUF in $\% / X_{0}$ ), is measured by lateral irradiation of the crystal using a $\mathrm{Co}^{60}$ source.

Due to the tapered geometry of the crystal, a polished crystal has an intrinsic non-uniform light collection along the crystal, i.e., the light yield measured at different positions along the crystals is not constant. To obtain a uniform light collection in the front region of the crystal, one of the lateral faces is de-polished. The required FNUF is obtained by adjusting the roughness value of the de-polishing on this face (see also the mechanical specifications) [16], [17].

Fig. 4 shows the effect of the roughness value of the depolished face on the front light collection non uniformity.

It has been observed that depolishing one lateral face induces a light yield loss of about 2 p.e./MeV compared to fully polished crystals.

Fig. 5 shows the front light collection non uniformity obtained for the BTCP and SIC crystals, respectively. 
TABLE III

OPTICAL TRANSMISSION SPECIFICATIONS

\begin{tabular}{ll}
\hline Parameters & Specification values \\
\hline Transversal transmission (TT) dispersion & $\Delta \lambda @$ TT $=50 \% \leq 3 \mathrm{~nm}$ for BTCP, $\leq 4 \mathrm{~nm}$ for SIC \\
Longitudinal transmission (LT) @ $\lambda=620 \mathrm{~nm}$ & LT $\geq 65 \%$ \\
$@ \lambda=420 \mathrm{~nm}$ & LT $\geq 55 \%$ \\
$@ \lambda=350 \mathrm{~nm}$ & LT $\geq 25 \%$ for BTCP \\
& LT $\geq 23 \%$ for SIC \\
Slope at the inflection point of the transmission curve & $>3 \% / \mathrm{nm}$ for BTCP only \\
& \\
\hline
\end{tabular}
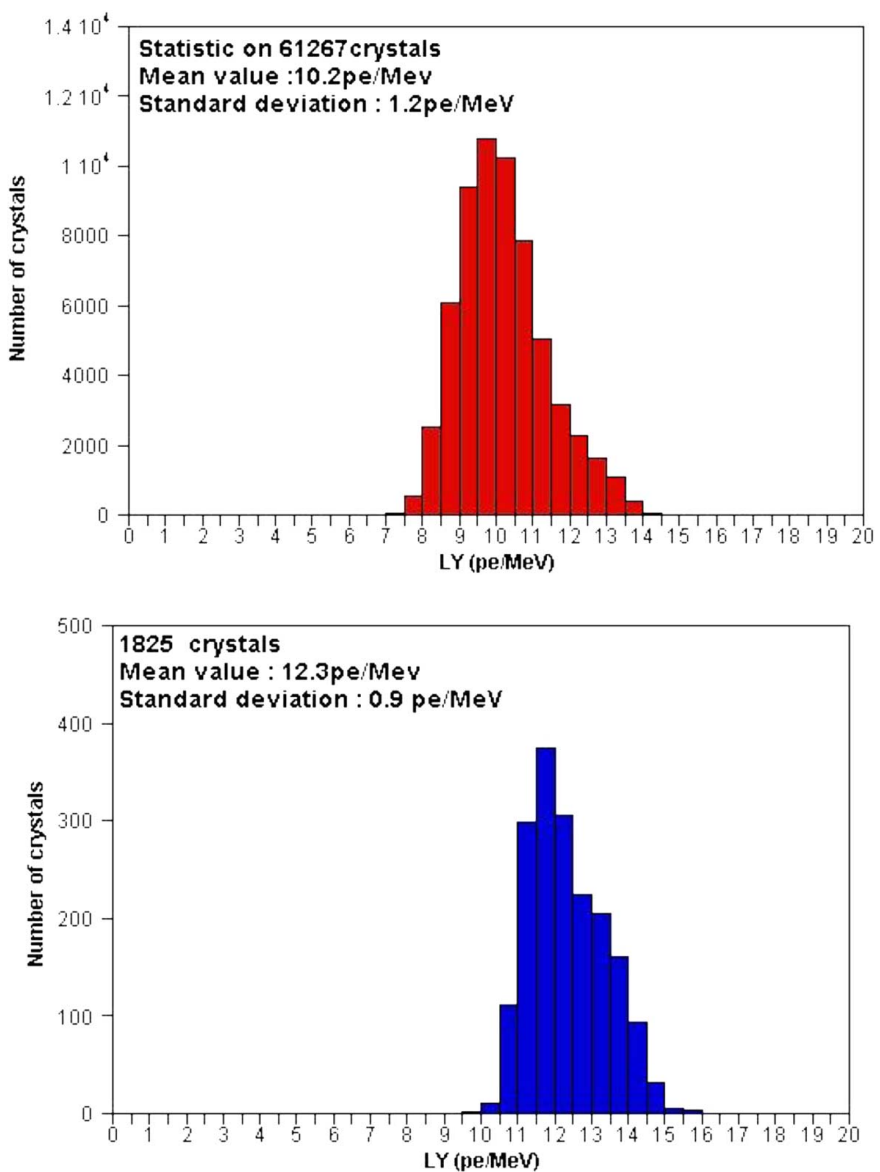

Fig. 6. Distribution of the Light Yield for (top) BTCP and (bottom) SIC crystals.

Crystals outside the specification limits were recovered at CERN by polishing (for FNUF $\leq-0.4 \% / X_{0}$ ) or lapping (for FNUF $\geq 0.4 \% / X_{0}$ ) the de-polished face.

The light yield distribution for the BTCP and SIC respectively crystals is shown in Fig. 6.

Almost all crystals from both producers are above the specification limit of 8 p.e./MeV.

SIC crystals have systematically a higher light yield of about 2 p.e./MeV than BTCP crystals (LY mean value of 12.3 p.e./MeV for SIC crystals, 10.2 p.e./MeV for BTCP).

\section{Optical Transmission}

The optical transmission is measured both longitudinally and transversally at different points along the crystal.

The detailed specifications for optical transmission properties are shown in Table III.
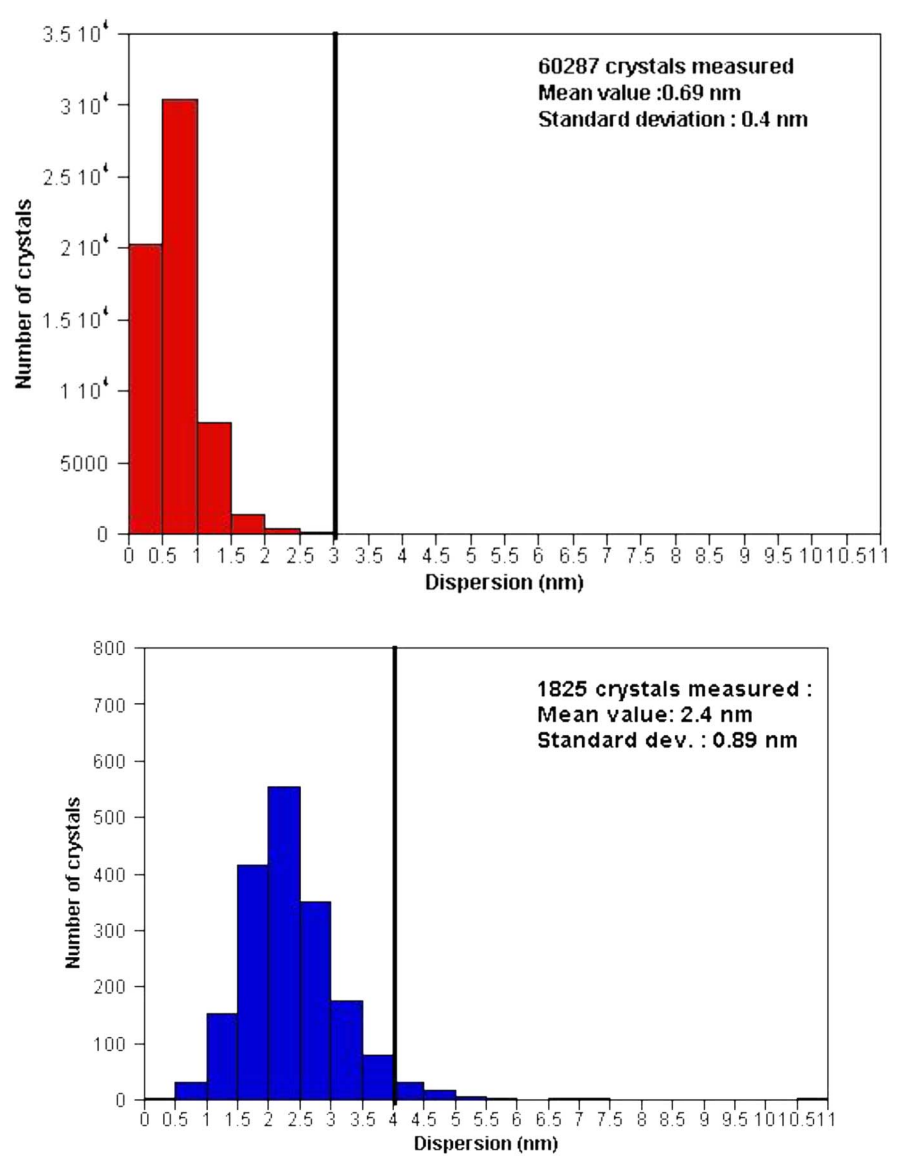

Fig. 7. Dispersion of the transversal transmission for (top) BTCP and (bottom) SIC crystals.

1) Transversal Transmission: A uniform transversal transmission is crucial for LY and radiation damage uniformity, both having a strong impact on the constant term of the energy resolution. This uniformity is quantified by the largest difference of the wavelengths at which the transversal transmission is $50 \%$ for the different measurement points (TT dispersion). Fig. 7 shows the TT dispersion obtained for the BTCP and SIC crystals. It is small for all BTCP crystals. For SIC crystals larger dispersion of transversal transmission were observed (probably correlated due to the growing technique), however, for most of these crystals this parameter is within the specifications limits.

2) Longitudinal Transmission: The longitudinal transmission is measured at 3 characteristic wavelengths:

- $620 \mathrm{~nm}$ : to detect the possible presence of core defects inside the crystals;

- $420 \mathrm{~nm}$ : to detect the eventual absorption band in the emission wavelength of PWO region. 

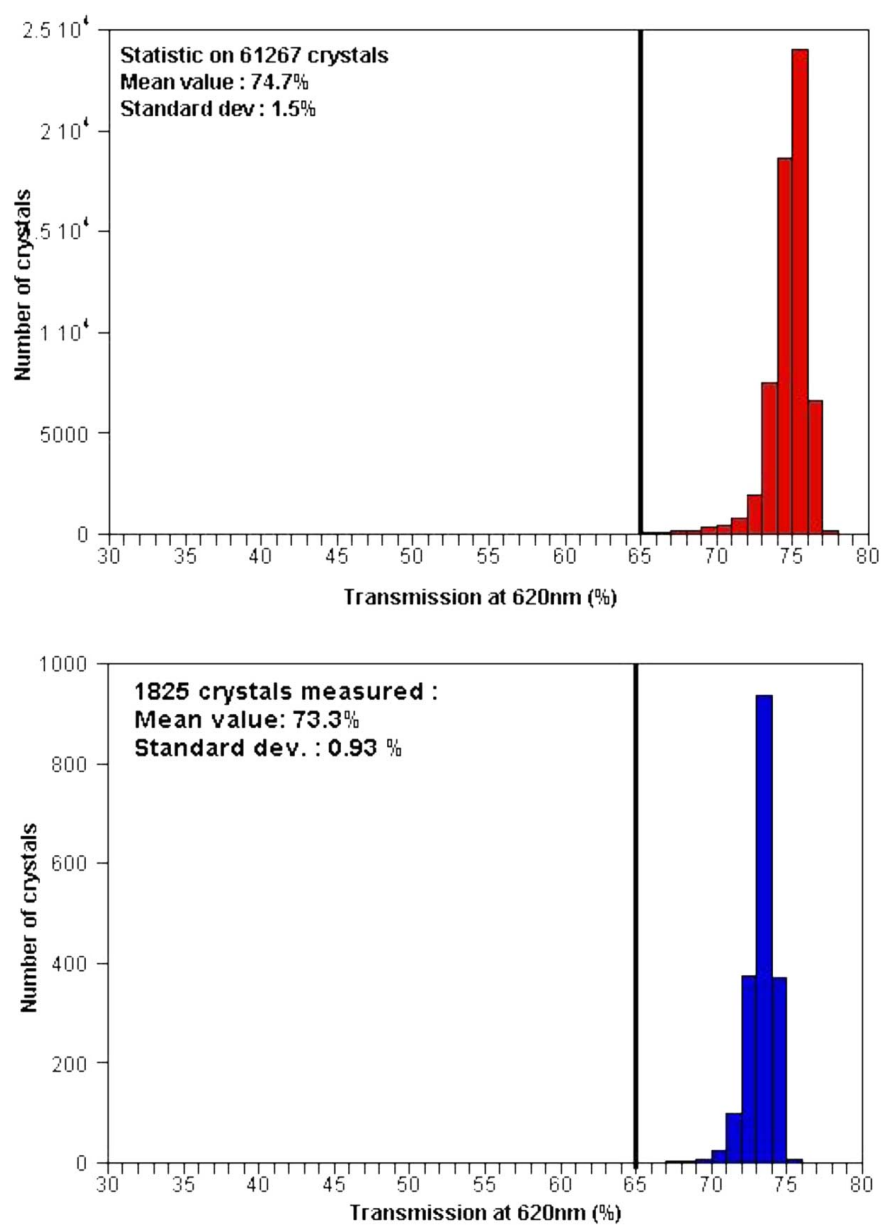

Fig. 8. Distribution of longitudinal transmission at $620 \mathrm{~nm}$ for (top) BTCP and (bottom) SIC crystals.

- $360 \mathrm{~nm}$ and the transmission slope in band-edge region (between 340 and $370 \mathrm{~nm}$ ): for radiation hardness prediction.

Fig. 8 shows the longitudinal transmission distribution at $620 \mathrm{~nm}$. All values are clearly above the specification limit both for BTCP and SIC, thus indicating the absence of core defects.

The longitudinal transmission at $420 \mathrm{~nm}$ is shown in Fig. 9. All crystals show a transmission value well above the specification limit. On average, the transmission is $4 \%$ smaller for SIC crystals than for BTCP crystals.

Fig. 10 shows the measurements for the longitudinal transmission at $360 \mathrm{~nm}$. Most crystals show a transmission value above the specification limit. The crystals below the specifications were systematically irradiated to control their radiation hardness.

The variation of the transmission in the distribution of the transmission values at $360 \mathrm{~nm}$ for BTCP crystals [Fig. 10 (top)], corresponds to a variation of the raw material used over 9 years of crystal production. After the preproduction phase, the doping was changed from $\mathrm{La}$ to $\mathrm{Y}-\mathrm{Nb}$ in order to improve radiation hardness [10]. The small shoulder at higher transmission is due to a trial of using raw material recycled from crystal cutting of previous production runs. The resulting higher transmission at $360 \mathrm{~nm}$ led to an increased light yield as shown in Fig. 11. However, it also resulted in a reduced radiation hardness due to a bad oxygen vacanciecompensation.
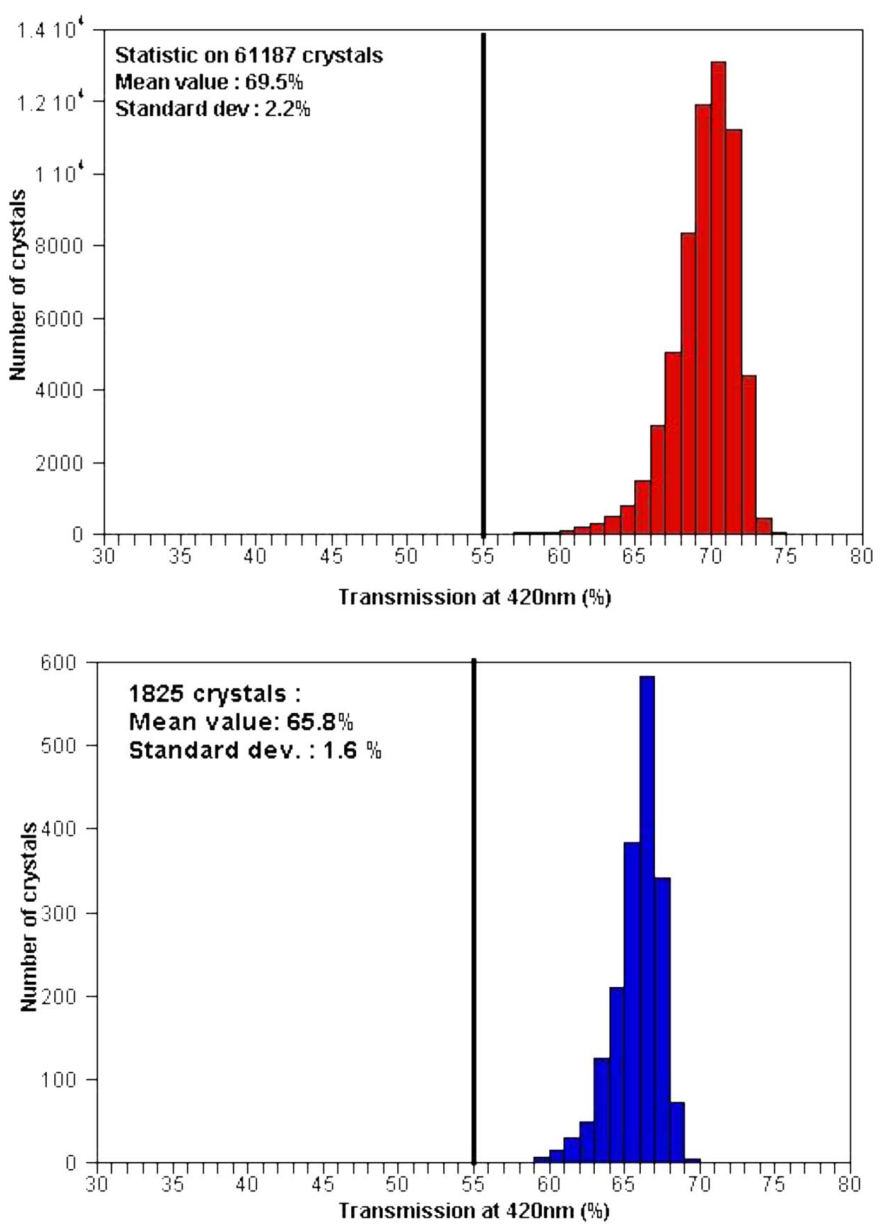

Fig. 9. Distribution of longitudinal transmission at $420 \mathrm{~nm}$ for (top) BTCP and (bottom) SIC crystals.

Fig. 11 shows the correlation between LY and LT360 nm. A correlation exists for both producers, but the correlation slope differs.

\section{Radiation Tolerance}

Radiation tolerance is an essential property for precision calorimetry at LHC. The radiation damage can have a strong impact on the light yield and the light collection uniformity and thus on the energy resolution. To limit the effects due to radiation under LHC conditions, additional specifications have been included and are shown in the Table IV.

Crystal samples from both SIC and BCTP were tested under irradiation either at high dose rate $(350 \mathrm{~Gy} / \mathrm{h})$ or under conditions comparable to those in the $\mathrm{LHC}(0.15 \mathrm{~Gy} / \mathrm{h})$. Fig. 11 shows the LY loss under frontal irradiation at LHC radiation levels $(0.15 \mathrm{~Gy} / \mathrm{h})$ for a sample of $1203 \mathrm{BCTP}$ crystals and 96 SIC crystals. The average light yield loss is $2.4 \%$ for BTCP crystals and $1.6 \%$ for SIC crystals well below the specifications.

The small loss in light yield will be corrected during the detector operation thanks to a monitoring system using laser light injected by optical fibers [18].

\section{CONCLUSION}

The choice of lead tungstate for electromagnetic calorimeter in 1994 was a challenge. The large R\&D effort performed over 5 years (1994 to 1998) by many institutes to understand the 
TABLE IV

RADIATION TOLERANCE SPECIFICATIONS

\begin{tabular}{ll}
\hline Parameters & Specification values \\
\hline $\begin{array}{l}\text { Induced absorption for full crystal saturation } \\
\text { (lateral irradiation, dose rate } \geq 30 \mathrm{~Gy} / \mathrm{h}\end{array}$ & $\mu \leq 1.5 \mathrm{~m}^{-1}$ at $\lambda=420 \mathrm{~nm}$ \\
$\begin{array}{l}\text { LY loss at LHC conditions } \\
\text { (frontal irradiation, dose rate }: 0.15 \mathrm{~Gy} / \mathrm{h} \text { ) }\end{array}$ & Light loss $\leq 6 \%$
\end{tabular}
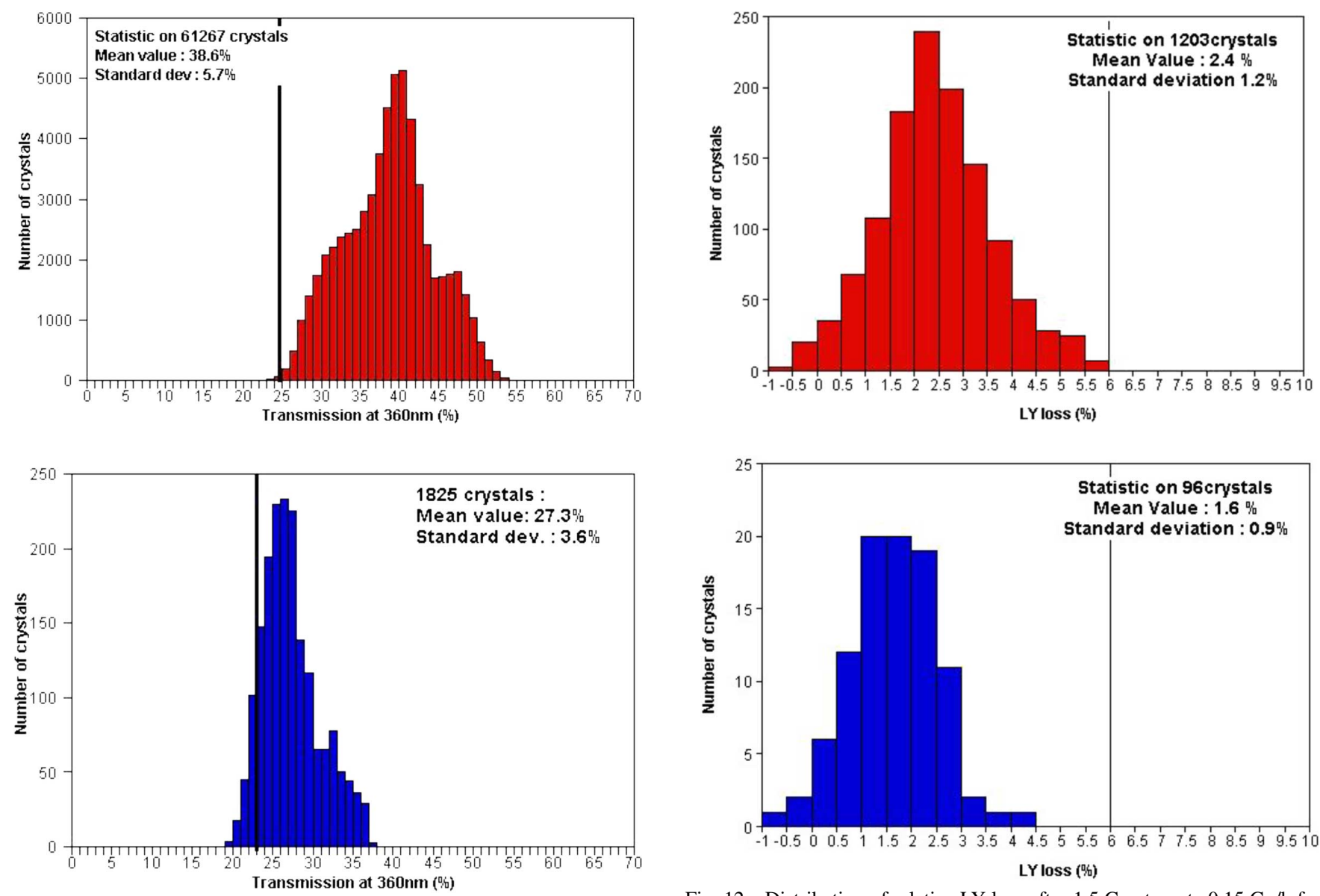

Fig. 10. Distribution of transmission at $360 \mathrm{~nm}$ for (top) BTCP and (bottom) SIC crystals.

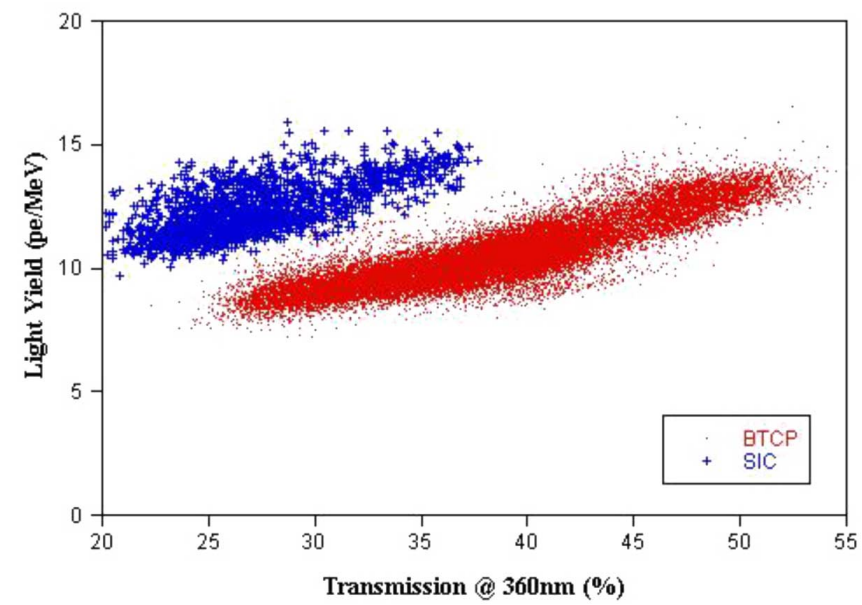

Fig. 11. Correlation between Light yield and transmission at $360 \mathrm{~nm}$.

fundamental properties of the crystals allowed us to improve the optical properties and radiation hardness of PWO to a level

Fig. 12. Distribution of relative LY loss after $1.5 \mathrm{~Gy}$ at a rate $0.15 \mathrm{~Gy} / \mathrm{h}$ for a sample of (top) BTCP and (bottom) SIC crystals.

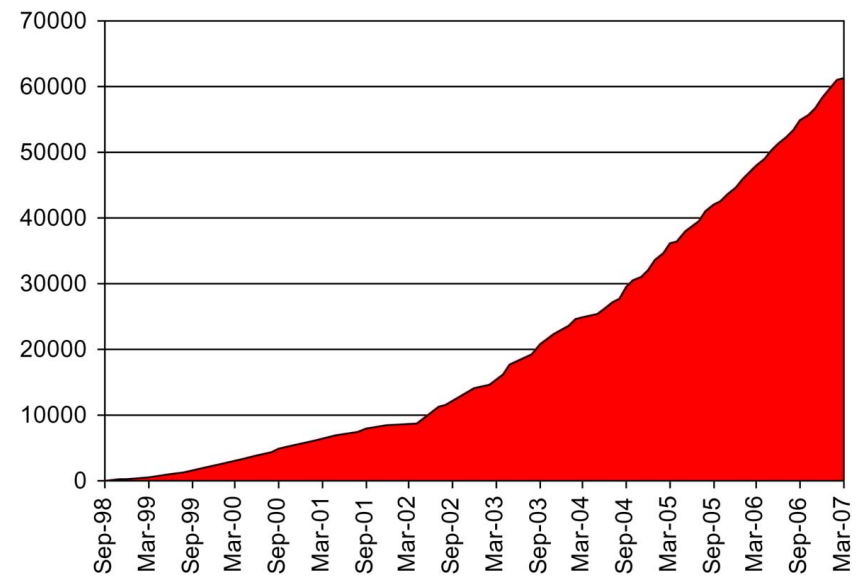

Fig. 13. Production rate of the BTCP crystals from 1998 to 2007.

required by the operational conditions of the LHC and consequently to launch a mass production. The development and optimisation of the production during the last 10 years enabled to 
reach a production rate of more than 1100 crystals per month (see Fig. 13) with a very low rejection rate.

A homogeneous quality level in terms of light yield, optical transmission and radiation tolerance has been achieved leading to a high performance detector. Tests with electron beams on several supermodules, each comprising 1700 crystals, have confirmed that the expected energy resolution of $0.5 \%$ at energies above $100 \mathrm{GeV}$ is obtained [19].

\section{ACKNOWLEDGMENT}

The author would like to thank all members of the CMS_ECAL group and all the scientific collaborators who contributed to the understanding and the improvement of lead tungstate properties.

\section{REFERENCES}

[1] The Compact Muon Solenoid Tech. Proposal, 1994, CERN/LHCC 94-38, LHCCC/P1, 15.

[2] The CMS ECAL Coll, 1997, Tech. Design Rep., CERN/LHCC 97-33.

[3] V. G. Barishevsky et al., "Single crystals of tungsten compounds as promising materials for the total absorption detectors of the E.M. calorimeters," Nucl. Instrum. Meth. A, vol. 322, pp. 231-231, 1992.

[4] M. Kobayashi, M. Ishii, and Y. Usuki, " $\mathrm{PbWO}_{4}$ scintillator at room temperature," in Proc. Crystal 2000 Int. Workshop, Gid-sur-Yvette, France, 1993, pp. 375-375.

[5] L. Nagornaya and V. Ryzhikov, "Fast scintillators based on large heavy tungstate single crystals," in Proc. Crystal 2000 Int. Workshop, Gif-surYvette, France, 1993, pp. 367-367.
[6] V. A. Kachanov et al., "Study of characteristics of real size $\mathrm{PbWO}_{4}$ crystal cells for precise EM-calorimeters to be used at LHC energies," in Proc. Crystal 2000 Int. Workshop, Gif-sur-Yvette, France, 1993, pp. 381-381.

[7] L. V. Miassoedov et al., "Heavy, fast and radiation resistant scintilator for EM calorimetry," in Proc. Crystal 2000 Int. Workshop, Gifsur-Yvette, France, 1993, pp. 367-367.

[8] E. Auffray et al., "Progress in the radiation hardness of PWO scintillators for the CMS calorimeter," in Proc. Int. Conf. Inorganic Scintillators and their Applications, Shanghai, China, 1997, pp. 219-219.

[9] A. Annenkov et al., "Suppression of the radiation damage in lead tungstate scintillation crystal," Nucl. Instrum. Meth. A, vol. 426, pp. 486-490, 1999.

[10] A. Annenkov et al., "Lead tungstate scintillation material," Nucl. Instrum. Meth. A, vol. 490, pp. 30-30, 2002.

[11] E. Auffray et al., "Status of the PWO crystal production from Russia for CMS-ECAL," Nucl. Instrum. Meth. A, vol. 486, pp. 111-111, 2002.

[12] E. Auffray et al., CMS Note 98/038.

[13] E. A. Auffray et al., "Performance of ACCOS, an automatic crystal quality control system for the PWO crystals of the CMS calorimeter," Nucl. Instrum. Meth. A, vol. 456, pp. 325-325, 2001.

[14] G. Basti et al., A Proposal for an Automatic Crystalscontrol System, CMS IN 97/033.

[15] E. Auffray et al., "Cross-calibration of two automatic quality control systems for the CMS ECAL crystals," Nucl. Instrum. Meth. A, vol. 523, pp. 355-355, 2004.

[16] E. Auffray et al., in Proc. Int. Workshop on Tungstate Crystals, Rome, Italy, 1999, pp. 275-275.

[17] E. Auffray et al., "Crystal conditioning for high-energy physics detectors," in Nucl. Instrum. Meth. A, 2002, vol. 486, pp. 22-22.

[18] J. Rander, "The monitoring system for the CMS electromagnetic calorimeter," in Proc. 8th Int. Conf. Calorimetry in High Energy Physics, Lisbon, Portugal, 1999, pp. 259-259.

[19] P. Adzic et al., "Results of the first performance tests of the cms electromagnetic calorimeter," Eur. Phys. J. C, vol. 44, pp. 1-25, 2006. 